\title{
Effects of isospin and energy dependences of the nuclear mean field in spallation reactions
}

\author{
Th. Aoust ${ }^{1,2, a}$ and J. Cugnon ${ }^{2, b}$ \\ 1 SCK-CEN, Boeretang 200, B-2400 Mol, Belgium \\ 2 University of Liège, Institute of Physics B5, Sart Tilman, B-4000 Liège 1, Belgium
}

Received: 28 October $2003 /$

Published online: 13 July 2004 - C Società Italiana di Fisica / Springer-Verlag 2004

Communicated by G. Orlandini

\begin{abstract}
In many applications of the intranuclear-cascade (INC) model to spallation reactions, all nucleons in the target are assumed to move in a common potential well. However, the potential depth should depend upon nucleon isospin and energy. The present paper describes the first results obtained after the introduction of these features in the Liège INCL3 model. It is shown that such modifications change cascade particle multiplicities significantly but total particle multiplicities are only slightly altered. Nucleon inclusive cross-sections are not modified significantly, except in the region of the quasi-elastic peaks. In particular, the centroid of the peak in neutron double differential cross-sections relative to proton-induced reactions can be sizeably shifted toward larger energy losses, as is observed experimentally. Implications of these results are discussed.
\end{abstract}

PACS. 25.40.-h Nucleon-induced reactions - 24.10.-i Nuclear reaction models and methods - 24.10.Lx Monte Carlo simulations (including hadron and parton cascades and string breaking models)

\section{Introduction}

There is a renewed interest in spallation reactions in the $\mathrm{GeV}$ range, mainly induced by several projects around the world concerning transmutation of nuclear wastes in accelerator-driven sytems (ADS) or other devices. The most successful tool for the description of these reactions is the intranuclear cascade (INC) plus evaporation model, which pictures the reaction mechanism as a first stage of well-separated successive nucleon-nucleon collisions followed by an evaporation stage. Recently, the Liège INC model [1] supplemented by the K.-H. Schmidt evaporation model $[2,3]$ has been shown to be very successful in the confrontation with a large set of experimental data for nucleon-induced spallation reactions in the $100 \mathrm{MeV}-2 \mathrm{GeV}$ range. However, this study presents some puzzling results. First, the description of the so-called quasi-elastic peak, which is largely dominated by single scattering, is generally less satisfactory than the one of the rest of the cross-section, which arises from multiple scattering. Second, the position of the quasi-elastic peak is well reproduced in the $p+A \rightarrow p+X$ inclusive cross-

\footnotetext{
a e-mail: taoust@sckcen.be

b e-mail: cugnon@plasma.theo.phys.ulg.ac.be
}

section and not in the $p+A \rightarrow n+X$ one $^{1}$. This might be attributed to using the same average potential well for protons and neutrons. Furthermore, this potential is supposed to be independent of the energy of the particles; this contradicts the common knowledge, accumulated from optical-model studies, which indicates that the real part of the optical-model potential shows an important momentum dependence. This potential, whose depth is around $50 \mathrm{MeV}$ for low-energy incident particles, practically vanishes when their incident energy exceeds $\sim 200 \mathrm{MeV}$. This might have some non-negligible effect on the neutron multiplicity, an important feature for transmtutation studies.

We want here to investigate the effects of the isospin and energy dependences of the nuclear-potential well, which are usually neglected in INC models. Actually, some version of the ISABEL model [4] has introduced these aspects, to some extent, but as far as we know, there is no systematic investigation of their effects. We present here such a study, using the Liège INC model. We will use the version named INCL3 [5], which basically differs from the one used in ref. [1] by the neglect of the diffuseness, because the present work has started before the last one has been finalized. Since we are mainly interested in showing

\footnotetext{
${ }^{1}$ Hereafter, we simply refer to these processes as $(p, p)$ and $(p, n)$ scatterings and the expression "quasi-elastic peak" applies to both processes.
} 
the variation for the results due the introduction of the isospin and energy dependences, the differences between the two models are not expected to play a significant role.

The paper is organized as follows. In sect. 2 we shortly present the INC model and the ingredients concerning the nuclear potential. We present results for particle multiplicities and nucleon double differential cross-sections in sect. 3 and discuss their implications. Section 4 contains our conclusion.

\section{The INC model and the average nuclear field}

\subsection{Reminder of the Liège INC model}

The version of the Liège INC model that we use here is described in detail in ref. [5]. It is sufficient here to recall that the nucleon-nucleus interaction process is described by a sequence of binary collisions occuring as in free space, except for Pauli blocking (here a strict Pauli blocking is applied instead of a statistical implementation as in ref. [5], but this is of no importance for the energies investigated here). At the beginning, target nucleons are distributed at random in the nuclear volume and their momenta are distributed at random in a Fermi sphere of radius $k_{\mathrm{F}}$. They are moving in an attractive potential of depth $V_{0}$. Nucleons can be excited, owing to collisions, to $\Delta$-resonances, which can decay into a pion and a nucleon. Of course inverse processes are also included. $\Delta$-resonances are supposed to experience the same potential as nucleons. $\mathrm{Pi}-$ ons are assumed not to feel any potential, for simplicity. This choice was basically motivated by the large uncertainties on the real part of the pion-nucleus optical-model potential [6]. The cascade process is terminated according to a physics criterion, which mainly reflects the end of the rapid variation of the target excitation energy, corresponding to the emission of fast particles. The further de-excitation of the target is handled by an evaporationfission code (here the Dresner code [7] is used). In the following we describe how we have implemented the isospin and energy dependences of the nuclear mean field. In order to enable us to disentangle the respective effects of the two features, we implemented them successively.

\subsection{Isospin dependence}

We consider that neutrons and protons are moving in potentials differing by their respective depths, $V_{0}^{i}$ for $i=n, p$. The quantities $V_{0}^{i}$ are determined as follows. Let $k_{\mathrm{F}}^{n}$ and $k_{\mathrm{F}}^{p}$ be the neutron and proton Fermi momenta (defining the sizes of the neutron and proton Fermi seas, respectively), and $T_{\mathrm{F}}^{i}$ the corresponding Fermi kinetic energies ${ }^{2}$

$$
T_{\mathrm{F}}^{i}=\frac{\hbar^{2} k_{\mathrm{F}}^{{ }^{2}}}{2 M},
$$

\footnotetext{
${ }^{2}$ Relativistic kinematics is used, but, for simplicity, we write
} down non-relativistic formulae.
Table 1. Fermi momenta and nuclear-potential depths for ${ }^{208} \mathrm{~Pb}$ and ${ }^{56} \mathrm{Fe}$ nuclei.

\begin{tabular}{|c|c|c|}
\hline & $\begin{array}{c}k_{\mathrm{F}}^{i} \\
(\mathrm{MeV} / c)\end{array}$ & $\begin{array}{c}V_{0}^{i} \\
(\mathrm{MeV}) \\
\end{array}$ \\
\hline No isospin dependence & 270.34 & 45.0 \\
\hline \multicolumn{3}{|l|}{ Isospin dependence } \\
\hline${ }_{82}^{208} \mathrm{~Pb}$ protons & 249.75 & 40.69 \\
\hline neutrons & 288.20 & 50.27 \\
\hline protons & 263.74 & 47.56 \\
\hline neutrons & 276.63 & 51.13 \\
\hline
\end{tabular}

where $M$ is the nucleon mass (we do not distinguish between neutron and proton). In accordance with the Fermi gas model $[8,9]$, the following relations are assumed:

$$
\begin{gathered}
\left(\frac{k_{\mathrm{F}}^{n}}{k_{\mathrm{F}}^{p}}\right)^{3}=\frac{N}{Z}, \\
\frac{1}{3 \pi^{2}}\left[\left(k_{\mathrm{F}}^{n}\right)^{3}+\left(k_{\mathrm{F}}^{p}\right)^{3}\right]=\rho_{0} .
\end{gathered}
$$

These relations, where $N$ and $Z$ are the target neutron and proton numbers, respectively, and $\rho_{0}$ is the normal nuclear matter density, determine the Fermi momenta uniquely. We now require that, in the spirit of Koopman's theorem [10], the total Fermi energy equals the separation energy, both for protons and neutrons:

$$
T_{\mathrm{F}}^{i}-V_{0}^{i}=-S_{i}
$$

The quantities $S_{i}$ 's can be taken from experiment (here values of ref. [11] are used) and the depth of the potentials can be determined from the last equation. Typical values are given in table 1 .

We can, of course, always write $V_{0}^{i}$ as

$$
V_{0}^{i}=V_{1}+V_{2} \tau_{i},
$$

where $\tau_{i}$ is twice the third component of the isopsin of the nucleons. For $\Delta$-particles we adopt the same form. In other words, the potential seen by the $\Delta^{+}\left(\Delta^{0}\right)$ particles is the same as the one felt by the protons (neutrons). There is no clear experimental information about the potential seen by the $\Delta$-particles, but this choice seems reasonable. We have to mention that theoretical evaluations [12] point to a somehow smaller value of $V_{1}$ for the $\Delta$ 's than for the nucleons. We keep however the same value, for simplicity.

It is interesting to establish a connection with the phenomenology of the optical-model potential. Let us call $k_{\mathrm{F}}$ the quantity defined by

$$
\frac{2}{3 \pi^{2}}\left(k_{\mathrm{F}}\right)^{3}=\rho_{0},
$$

i.e. the average Fermi momentum and let us suppose that $S_{p}=S_{n}(=S)$, for simplicity. In ref. [5], where the same Fermi momentum was taken for neutrons and protons and the equality of separation energy was assumed, $k_{\mathrm{F}}=270 \mathrm{MeV} / c$ and $V_{0}^{i}=V_{0}=45 \mathrm{MeV}$. Using eqs. (2), (3) and (6), one writes

$$
k_{\mathrm{F}}^{i}=k_{\mathrm{F}}\left(1-\xi \tau_{i}\right)^{1 / 3},
$$


where $\xi$ is given by

$$
\xi=\frac{N-Z}{A}=-\frac{2 T_{z}}{A}
$$

The quantity $T_{z}$ is the $z$-component of the target isospin. With the help of eq. (4), one gets:

$$
V_{0}^{i}=\frac{\hbar^{2} k_{\mathrm{F}}^{2}}{2 M}\left(1-\xi \tau_{i}\right)^{2 / 3}+S
$$

Expanding expression (9) to first order in $\xi$ (it is easy to see that non-linear terms in $\xi$ are small, even for heavy nuclei), we obtain expression (5) with

$$
V_{1} \approx \frac{\hbar^{2} k_{\mathrm{F}}^{2}}{2 M}+S
$$

and

$$
V_{2} \approx-\frac{2}{3} \frac{\hbar^{2} k_{\mathrm{F}}^{2}}{2 M} \xi
$$

In optical-model phenomenology, it is customary to write down the proton and neutron average potentials as [13]

$$
V_{0}^{p}=U_{0}+U_{1} \xi+0.4 \frac{Z}{A^{1 / 3}}-\overline{V_{\mathrm{c}}},
$$

where the third term is the so-called Coulomb anomaly and where $\overline{V_{\mathrm{c}}}$ is the average Coulomb potential (in the nuclear volume), and

$$
V_{0}^{n}=U_{0}-U_{1} \xi
$$

Rewriting these expressions as in eq. (5) (using quantities with upper index ph to recall that they are defined phenomenologically), one has

$$
V_{1}^{\mathrm{ph}}=U_{0}+\frac{1}{2}\left(0.4 \frac{Z}{A^{1 / 3}}-\overline{V_{\mathrm{c}}}\right),
$$

and

$$
V_{2}^{\mathrm{ph}}=U_{1} \xi+\frac{1}{2}\left(0.4 \frac{Z}{A^{1 / 3}}-\overline{V_{\mathrm{c}}}\right) .
$$

Although the dependence upon $\xi$ is not the same, the quantities appearing in eqs. (14), (15) are numerically close to those appearing in eqs. (10), (11). For instance, for a ${ }^{208} \mathrm{~Pb}$ target, $V_{1} \approx 45 \mathrm{MeV}$ and $V_{2} \approx-5 \mathrm{MeV}$, to be compared with $V_{1}^{\text {ph }} \approx 40 \mathrm{MeV}$ and $V_{2}^{\text {ph }} \approx-2 \mathrm{MeV}$. For ${ }^{56} \mathrm{Fe}, V_{1} \approx 47 \mathrm{MeV}$ and $V_{2} \approx-1.8 \mathrm{MeV}$, to be compared with $V_{1}^{\mathrm{ph}} \approx 42 \mathrm{MeV}$ and $V_{2}^{\mathrm{ph}} \approx-1.0 \mathrm{MeV}$. Although the comparison is not perfect, it indicates that our simple parametrization of the $V_{0}^{i}$ 's is quite reasonable.

\subsection{Energy dependence}

It is well known that the nuclear mean field is momentum dependent. This property can also be formulated as an energy dependence [14]. Phenomenology tells that the depth of the nuclear potential decreases roughly linearly when the total energy increases until the latter reaches $\sim 200 \mathrm{MeV}$. The potential vanishes at this energy and is very small for larger energies. We will adopt here the following nucleon potential

$$
\begin{aligned}
V_{0}^{i}(E) & =V_{0}^{i}-\alpha_{i}\left(E-E_{\mathrm{F}}^{i}\right), \quad \text { for } \quad E<E_{0} \\
& =0, \quad \text { for } \quad E>E_{0},
\end{aligned}
$$

where $E$ is given by

$$
E=\frac{\hbar^{2} k^{2}}{2 M}+V_{0}^{i}(E)
$$

and where $E_{\mathrm{F}}^{i}$ is defined by the same expression with $k=$ $k_{\mathrm{F}}^{i}$. The quantity $E_{0}$ is the energy where the expression of the first line of eq. (16) vanishes. It is evident from the last two expressions that the Fermi (kinetic and total) energies remain the same as before introducing the energy dependence and that the new potentials are the same as the previous ones at the Fermi levels. Of course, eqs. (16), (17) allow to rewrite the potentials as functions of the momentum or of the kinetic energy of the particle. Below we use $\alpha_{p}=\alpha_{n}=0.23$, following ref. [15].

We will also consider potentials with a smoother energy dependence

$$
V_{0}^{i}(E)=V_{0}^{i} \exp \left(-\beta_{i}\left(E-E_{\mathrm{F}}^{i}\right)\right),
$$

with $\beta_{i}=0.00681$, as in refs. $[13,16]$

The energy dependence has been neglected for $\Delta$ particles, for simplicity. This seems reasonable, in view of the scarce theoretical information on the subject.

\subsection{Implementation in the INC code}

Let us consider first binary reactions $N_{1} N_{2} \rightleftharpoons N_{3} N_{4}$, where $N_{i}$ stands generically for nucleons and $\Delta$ 's. One has to fulfill energy conservation laws:

$$
\begin{gathered}
\mathbf{p}_{1}+\mathbf{p}_{2}=\mathbf{p}_{3}+\mathbf{p}_{4}, \\
E_{1}+V_{0}^{1}+E_{2}+V_{0}^{2}=E_{3}+V_{0}^{3}+E_{4}+V_{0}^{4},
\end{gathered}
$$

where the $E_{i}$ 's are the mass plus kinetic energies ${ }^{3}$. For a unique potential (no isospin dependence, no energy dependence, same potential for nucleons and $\Delta$ 's) as in the standard version of the Liège model, the potentials cancel from both sides of eq. (20). Note that they also cancel in the case of isospin-dependent potentials (eq. (5)) for nucleon-nucleon collisions.

For energy-dependent potentials, we proceed as follows. We transform the 4-momenta $\left(E_{i}, \mathbf{p}_{i}\right)$ in the center of mass frame of the colliding pair. We determine the final c.m. momentum $p *$ and the angles neglecting the potentials. The effects of the latter is simulated by multiplying $p *$ by a factor $f$. After transforming back in the original frame, eq. (19) is automatically verified and eq. (20)

\footnotetext{
${ }^{3}$ We consider here the nuclear potential as a vector potential, for simplicity. Since most of the particles involved have small kinetic energy, results are expected to be the same for another choice of the Lorentz character of the potential.
} 
Table 2. Multiplicities per primary reaction obtained in proton-induced reactions on ${ }^{208} \mathrm{~Pb}$ and ${ }^{56} \mathrm{Fe}$ nuclei at $800 \mathrm{MeV}$. Excitation energies $\left(E^{*}\right)$ are also given (in $\left.\mathrm{MeV}\right)$. Experimental data are from ref. [18].

\begin{tabular}{|c|c|c|c|c|c|c|c|}
\hline \multicolumn{8}{|c|}{$p(800 \mathrm{MeV})+{ }^{208} \mathrm{~Pb}$} \\
\hline & & & Exp. & Standard & Isospin & $\begin{array}{l}\text { Isospin } \\
\text { and energy } \\
\text { linear }\end{array}$ & $\begin{array}{c}\text { Isospin } \\
\text { and energy } \\
\text { exponential }\end{array}$ \\
\hline casc & $\begin{array}{l}n \\
\\
p \\
\\
{ }^{*} \\
E^{*}\end{array}$ & $\begin{array}{l}E>20 \mathrm{MeV} \\
20 \mathrm{MeV}>E>2 \mathrm{MeV} \\
2 \mathrm{MeV}>E \\
\text { total } \\
E>20 \mathrm{MeV} \\
E<20 \mathrm{MeV} \\
\text { total }\end{array}$ & & $\begin{array}{l}2.050 \\
0.613 \\
0.039 \\
2.702 \\
1.874 \\
0.177 \\
2.051 \\
0.419 \\
214.370\end{array}$ & $\begin{array}{c}1.918 \\
0.576 \\
0.036 \\
2.530 \\
1.959 \\
0.187 \\
2.146 \\
0.421 \\
217.580\end{array}$ & $\begin{array}{l}1.824 \\
0.444 \\
0.026 \\
2.294 \\
1.925 \\
0.150 \\
2.075 \\
0.390 \\
227.455\end{array}$ & $\begin{array}{c}1.784 \\
0.411 \\
0.023 \\
2.218 \\
1.939 \\
0.151 \\
2.090 \\
0.394 \\
241.504\end{array}$ \\
\hline $\begin{array}{c}\text { casc } \\
+ \text { evap }\end{array}$ & $\begin{array}{l}p \\
\pi \\
\alpha \\
d \\
t\end{array}$ & $\begin{array}{l}E>20 \mathrm{MeV} \\
20 \mathrm{MeV}>E>2 \mathrm{MeV} \\
2 \mathrm{MeV}>E \\
\text { total } \\
E>20 \mathrm{MeV} \\
E<20 \mathrm{MeV} \\
\text { total }\end{array}$ & $\begin{array}{l}1.9 \\
6.5\end{array}$ & $\begin{array}{c}2.103 \\
9.166 \\
5.163 \\
16.432 \\
1.903 \\
1.411 \\
3.314 \\
0.419 \\
0.911 \\
0.597 \\
0.402\end{array}$ & $\begin{array}{c}1.974 \\
9.316 \\
5.209 \\
16.499 \\
1.988 \\
1.410 \\
3.398 \\
0.421 \\
0.910 \\
0.605 \\
0.412\end{array}$ & $\begin{array}{c}1.887 \\
9.582 \\
5.286 \\
16.755 \\
1.957 \\
1.439 \\
3.396 \\
0.390 \\
0.963 \\
0.650 \\
0.446\end{array}$ & $\begin{array}{c}1.857 \\
10.07 \\
5.392 \\
17.319 \\
1.975 \\
1.540 \\
3.515 \\
0.394 \\
1.048 \\
0.722 \\
0.496\end{array}$ \\
\hline \multicolumn{8}{|c|}{$p(800 \mathrm{MeV})+{ }^{56} \mathrm{Fe}$} \\
\hline & & & Exp. & Standard & Isospin & $\begin{array}{l}\text { Isospin } \\
\text { and energy } \\
\text { linear }\end{array}$ & $\begin{array}{c}\text { Isospin } \\
\text { and energy } \\
\text { exponential }\end{array}$ \\
\hline casc & $\begin{array}{l}n \\
p \\
\\
\\
\pi^{*}\end{array}$ & $\begin{array}{l}E>20 \mathrm{MeV} \\
20 \mathrm{MeV}>E>2 \mathrm{MeV} \\
2 \mathrm{MeV}>E \\
\text { total } \\
E>20 \mathrm{MeV} \\
E<20 \mathrm{MeV} \\
\text { total }\end{array}$ & & $\begin{array}{c}1.575 \\
0.326 \\
0.019 \\
1.920 \\
1.938 \\
0.284 \\
2.222 \\
0.487 \\
118.497\end{array}$ & $\begin{array}{c}1.480 \\
0.303 \\
0.018 \\
1.801 \\
1.914 \\
0.279 \\
2.193 \\
0.487 \\
121.833\end{array}$ & $\begin{array}{c}1.447 \\
0.243 \\
0.014 \\
1.704 \\
1.902 \\
0.225 \\
2.127 \\
0.443 \\
131.789\end{array}$ & $\begin{array}{c}1.437 \\
0.231 \\
0.013 \\
1.681 \\
1.906 \\
0.221 \\
2.127 \\
0.446 \\
144.978\end{array}$ \\
\hline $\begin{array}{c}\text { casc } \\
+ \text { evap }\end{array}$ & $\begin{array}{l}p \\
\pi \\
\alpha \\
d \\
t\end{array}$ & $\begin{array}{l}E>20 \mathrm{MeV} \\
20 \mathrm{MeV}>E>2 \mathrm{MeV} \\
2 \mathrm{MeV}>E \\
\text { total } \\
E>20 \mathrm{MeV} \\
E>20 \mathrm{MeV} \\
\text { total }\end{array}$ & $\begin{array}{l}1.4 \\
1.7\end{array}$ & $\begin{array}{l}1.659 \\
2.607 \\
1.127 \\
5.393 \\
2.038 \\
2.659 \\
4.697 \\
0.487 \\
0.403 \\
0.410 \\
0.084\end{array}$ & $\begin{array}{l}1.570 \\
2.663 \\
1.141 \\
5.274 \\
2.017 \\
2.670 \\
4.687 \\
0.487 \\
0.412 \\
0.428 \\
0.089\end{array}$ & $\begin{array}{l}1.550 \\
2.790 \\
1.169 \\
5.509 \\
2.019 \\
2.761 \\
4.789 \\
0.443 \\
0.447 \\
0.479 \\
0.101\end{array}$ & $\begin{array}{l}1.563 \\
3.021 \\
1.201 \\
5.785 \\
2.046 \\
2.938 \\
4.984 \\
0.446 \\
0.488 \\
0.552 \\
0.117\end{array}$ \\
\hline
\end{tabular}

appears as an algebraic equation for $f$, that we solve iteratively. Generally $f$ is close to unity and a small number of iterations is sufficient. We proceed in the same manner for $\Delta$-decay. For the $\pi+N \rightarrow \Delta$ reactions, the procedure is simpler and does not require any iteration. Indeed, the energy momentum conservation equation writes, with the indices 1 for pion (no potential), 2 for nucleon and 3 for Delta,

$$
\begin{aligned}
\mathbf{p}_{1}+\mathbf{p}_{2} & =\mathbf{p}_{3}, \\
E_{1}+E_{2}+V_{0}^{2} & =E_{3}+V_{0}^{3},
\end{aligned}
$$

in the target frame and

$$
\begin{gathered}
\mathbf{p}_{1}^{\prime}+\mathbf{p}_{2}^{\prime}=0 \\
E_{1}^{\prime}+E_{2}^{\prime}+V_{0}^{2^{\prime}}=E_{3}^{\prime}+V_{0}^{3^{\prime}}
\end{gathered}
$$

in the pion-nucleon center-of-mass frame (with respect to the 4 -vectors $\left(E_{1}, \mathbf{p}_{1}\right)$ and $\left.\left(E_{2}, \mathbf{p}_{2}\right)\right)$. The primes indicate that the quantities have been transformed in this frame. Equation (24) determines $E_{3}^{\prime}$ (since all other quantities are known), which is nothing but the mass of the $\Delta$-particle. A Lorentz transformation back to the target frame allows the determination of the 4 -vector $\left(E_{3}, \mathbf{p}_{3}\right)$.

\section{Results}

We compare results obtained with the standard model and the modified model for the case of proton-induced reactions on ${ }^{208} \mathrm{~Pb}$ and ${ }^{56} \mathrm{Fe}$ nuclei, both at $256 \mathrm{MeV}$ and at $800 \mathrm{MeV}$. 
Table 3. Multiplicities per primary reaction obtained in proton-induced reactions on ${ }^{208} \mathrm{~Pb}$ and ${ }^{56} \mathrm{Fe}$ nuclei at $256 \mathrm{MeV}$. Excitation energies $\left(E^{*}\right)$ are also given (in $\mathrm{MeV}$ ).

\begin{tabular}{|c|c|c|c|c|c|c|}
\hline \multicolumn{7}{|c|}{$p(256 \mathrm{MeV})+{ }^{208} \mathrm{~Pb}$} \\
\hline & & & Standard & Isospin & $\begin{array}{l}\text { Isospin } \\
\text { and energy } \\
\text { linear }\end{array}$ & $\begin{array}{c}\text { Isospin } \\
\text { and energy } \\
\text { exponential }\end{array}$ \\
\hline casc & $\begin{array}{c}p \\
\pi^{*} \\
E^{*}\end{array}$ & $\begin{array}{l}E>20 \mathrm{MeV} \\
20 \mathrm{MeV}>E>2 \mathrm{MeV} \\
2 \mathrm{MeV}>E \\
\text { total } \\
E>20 \mathrm{MeV} \\
E<20 \mathrm{MeV} \\
\text { total }\end{array}$ & $\begin{array}{l}0.966 \\
0.507 \\
0.035 \\
1.508 \\
1.028 \\
0.143 \\
1.171 \\
0.029 \\
88.086\end{array}$ & $\begin{array}{c}0.876 \\
0.456 \\
0.033 \\
1.355 \\
1.108 \\
0.158 \\
1.266 \\
0.026 \\
90.226\end{array}$ & $\begin{array}{l}0.838 \\
0.360 \\
0.024 \\
1.222 \\
1.121 \\
0.135 \\
1.256 \\
0.012 \\
94.231\end{array}$ & $\begin{array}{l}0.806 \\
0.329 \\
0.021 \\
1.256 \\
1.136 \\
0.135 \\
1.261 \\
0.013 \\
99.455\end{array}$ \\
\hline $\begin{array}{c}\text { casc } \\
+ \text { evap }\end{array}$ & $\begin{array}{l}p \\
\pi \\
\alpha \\
d \\
t\end{array}$ & $\begin{array}{l}E>20 \mathrm{MeV} \\
20 \mathrm{MeV}>E>2 \mathrm{MeV} \\
2 \mathrm{MeV}>E \\
\text { total } \\
E>20 \mathrm{MeV} \\
E<20 \mathrm{MeV} \\
\text { total }\end{array}$ & $\begin{array}{l}0.968 \\
4.198 \\
3.750 \\
8.916 \\
1.030 \\
0.432 \\
1.462 \\
0.029 \\
0.123 \\
0.059 \\
0.033\end{array}$ & $\begin{array}{l}0.879 \\
4.272 \\
3.806 \\
8.957 \\
1.110 \\
0.448 \\
1.558 \\
0.026 \\
0.126 \\
0.061 \\
0.036\end{array}$ & $\begin{array}{l}0.841 \\
4.388 \\
3.890 \\
8.919 \\
1.123 \\
0.447 \\
1.570 \\
0.012 \\
0.138 \\
0.069 \\
0.040\end{array}$ & $\begin{array}{l}0.809 \\
4.611 \\
4.006 \\
9.426 \\
1.138 \\
0.476 \\
1.614 \\
0.013 \\
0.155 \\
0.079 \\
0.047\end{array}$ \\
\hline \multicolumn{7}{|c|}{$p(256 \mathrm{MeV})+{ }^{56} \mathrm{Fe}$} \\
\hline & & & Standard & Isospin & $\begin{array}{l}\text { Isospin } \\
\text { and energy } \\
\text { linear }\end{array}$ & $\begin{array}{c}\text { Isospin } \\
\text { and energy } \\
\text { exponential }\end{array}$ \\
\hline casc & $\begin{array}{c}p \\
\pi^{*} \\
E^{*}\end{array}$ & $\begin{array}{l}E>20 \mathrm{MeV} \\
20 \mathrm{MeV}>E>2 \mathrm{MeV} \\
2 \mathrm{MeV}>E \\
\text { total } \\
E>20 \mathrm{MeV} \\
E<20 \mathrm{MeV} \\
\text { total }\end{array}$ & $\begin{array}{l}0.830 \\
0.302 \\
0.020 \\
1.152 \\
1.232 \\
0.288 \\
1.520 \\
0.033 \\
56.982 \\
\end{array}$ & $\begin{array}{l}0.763 \\
0.274 \\
0.018 \\
1.055 \\
1.220 \\
0.284 \\
1.504 \\
0.033 \\
59.267 \\
\end{array}$ & $\begin{array}{l}0.750 \\
0.225 \\
0.014 \\
0.989 \\
1.213 \\
0.242 \\
1.455 \\
0.013 \\
64.556 \\
\end{array}$ & $\begin{array}{l}0.735 \\
0.213 \\
0.013 \\
0.961 \\
1.223 \\
0.236 \\
1.459 \\
0.015 \\
70.319 \\
\end{array}$ \\
\hline $\begin{array}{c}\text { casc } \\
+ \text { +evap }\end{array}$ & $\begin{array}{l}p \\
\pi \\
\alpha \\
d \\
t\end{array}$ & $\begin{array}{l}E>20 \mathrm{MeV} \\
20 \mathrm{MeV}>E>2 \mathrm{MeV} \\
2 \mathrm{MeV}>E \\
\text { total } \\
E>20 \mathrm{MeV} \\
E<2 \mathrm{MeV} \\
\text { total }\end{array}$ & $\begin{array}{l}0.836 \\
1.303 \\
0.871 \\
3.010 \\
1.244 \\
1.743 \\
3.087 \\
0.033 \\
0.186 \\
0.096 \\
0.014\end{array}$ & $\begin{array}{l}0.770 \\
1.334 \\
0.895 \\
2.999 \\
1.233 \\
1.761 \\
3.094 \\
0.033 \\
0.196 \\
0.104 \\
0.016\end{array}$ & $\begin{array}{l}0.759 \\
1.396 \\
0.924 \\
3.079 \\
1.229 \\
1.831 \\
3.062 \\
0.013 \\
0.223 \\
0.124 \\
0.020\end{array}$ & $\begin{array}{l}0.747 \\
1.512 \\
0.961 \\
3.220 \\
1.242 \\
1.936 \\
3.178 \\
0.015 \\
0.250 \\
0.145 \\
0.024\end{array}$ \\
\hline
\end{tabular}

\subsection{Particle multiplicities}

A thorough comparison is provided by tables 2 and 3. For each case, the results refer to a sample of two millions of events, allowing most of the times to detect variations of multiplicities of 0.01 beyond statistical uncertainty.

We first discuss the effect of the introduction of the isospin dependence. The most important result is the reduction of the cascade neutron multiplicity, especially for the $\mathrm{Pb}$ case. The reduction is proportionally more important at low incident energy. Correlatively, the proton cascade multiplicity is increased, in the $\mathrm{Pb}$ case, also more importantly at lower energy. In our opinion, this is a simple consequence of the modification of the Fermi momenta. Compared to the standard case, the proton Fermi momentum has been diminished, while the neutron Fermi momentum has increased. Since the Fermi energy (both for protons and neutrons) has basically remained the same as in the standard case, the average (single-particle) energy of the target protons is higher than the one of the target neutrons. It is therefore easier (more difficult) to remove a proton (a neutron) in the modified case than in the standard case. This argument also explains why the effect is smaller for Fe, which is almost charge symmetric. In this case, the proton cascade multiplicity is even slightly decreased, which arises from the fact that, due to the relatively small difference of the Fermi momenta, the difference in the separation energies (see table 1) also plays a role. Let us mention in passing that in the Fe case, the cascade proton multiplicity exceeds the neutron one. This is due to the dominant role of the incident proton, as explained in ref. [17].

It can be seen that the excitation energy is slightly more important with the modified potential. As a 
consequence, the number of evaporated particles is slightly increased. Globally, the total neutron multiplicity is only very slightly increased for $\mathrm{Pb}$ and basically remains untouched for Fe. The total proton multiplicity roughly follows the same pattern. The multiplicities of other evaporated particles are moderately changed and follow the modifications of the excitation energy.

When the (linear) energy dependence is added, the tendency for the cascade neutron multiplicity is enhanced: it is further decreased and the total reduction in comparison with the standard case reaches $10-15 \%$ at $800 \mathrm{MeV}$ and $20 \%$ at $256 \mathrm{MeV}$. As for the protons, their multiplicity is slightly decreased by the introduction of the energy dependence. In our opinion, the physical origin of these effects is the following: since the potential energy is increasing linearly (in absolute value) below the Fermi level (see eq. (16)), the average single-particle energy of the bound nucleons is lowered, compared to the case with the isospin-dependent and energy-independent potential. The lowering is more important for the neutrons than for the protons, as the Fermi kinetic energy is larger for the first kind of particles. The comparison between the standard case and the isospin- and energy-dependent potential case reveals that the proton cascade multiplicity is larger in the second case, for the $\mathrm{Pb}$ target, while it is smaller for Fe target.

With the introduction of the linear energy dependence, the excitation energy is further increased. The total increase reaches $6 \%$ for $\mathrm{Pb}$ at $800 \mathrm{MeV}$. The trends are accentuated: this time, the total neutron multiplicity is definitely, though slightly (with respect to the standard case), increased for $\mathrm{Pb}$ at both energies. Note, however, that the neutron multiplicity for $E>20 \mathrm{MeV}$ is substantially decreased. The modification of the total proton multiplicity shows no definite pattern. The total increase of the proton multiplicity, compared to the standard case, is of the order of $2 \%$ at $800 \mathrm{MeV}$ and of $8 \%$ at $256 \mathrm{MeV}$ for $\mathrm{Pb}$, but practically vanishes for $\mathrm{Fe}$ at the same energy. In the $E>20$ $\mathrm{MeV}$ range, the proton multiplicity is sizeably increased.

Finally let us notice that the results are basically the same when an exponential energy dependence is adopted for the nuclear mean field (last columns of tables 2 and $3)$, but the trends are more pronounced.

\subsection{Particle spectra}

A comparison is made in figs. 1 and 2. Concerning neutron spectra (fig. 1), the most important result is the shift of the quasi-elastic peak toward lower energy when the isospin dependence is introduced, a shift which is amplified when the energy dependence is further introduced. The difference between the two choices of energy dependences (eqs. (16), (18)) is not very significant except at 0 degrees. The total shift amounts to $\sim 15 \mathrm{MeV}$ at 0 degrees and reaches $\sim 35 \mathrm{MeV}$ at 10 degrees, which goes in the good direction if one compares with the experimental cross-section. There is also a slight increase of the intensity of the quasi-elastic peak, which also helps to cure the

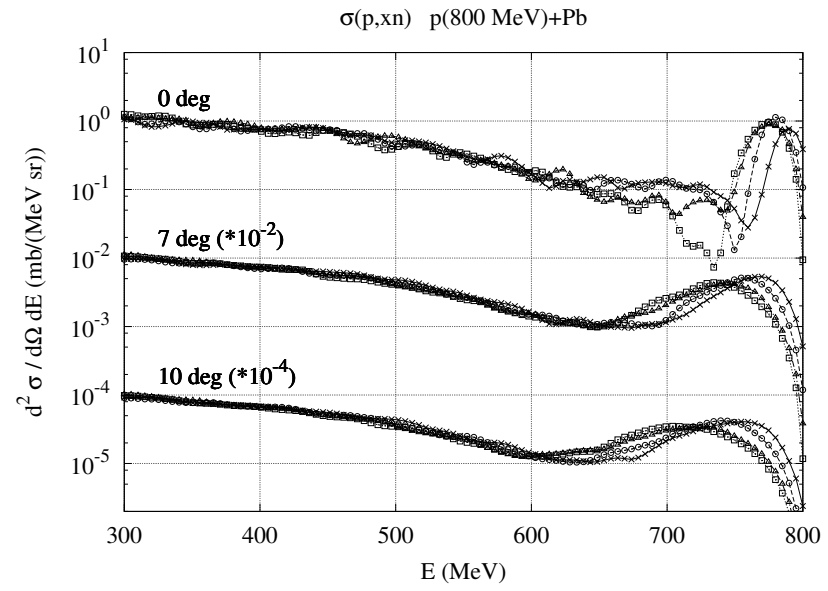

Fig. 1. Neutron double differential cross-section for protoninduced reactions on ${ }^{208} \mathrm{~Pb}$ at $800 \mathrm{MeV}$. Comparison of theoretical results with different choices of the nuclear average potential: standard choice, isospin- and energy-independent potential (full line with crosses), isospin-dependent, energyindependent potential (dashed line with circles), isospinand linearly energy-dependent potential (eq. (16)) (dashdotted line with triangles), isospin- and exponentially energydependent potential (eq. (18)) (dotted line with squares).

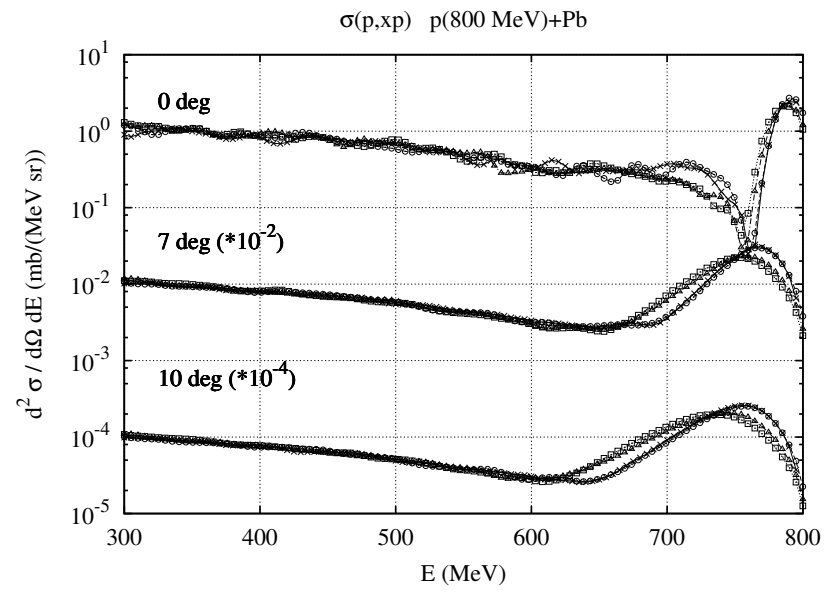

Fig. 2. Proton double differential cross-section for protoninduced reactions on ${ }^{208} \mathrm{~Pb}$ at $800 \mathrm{MeV}$. Comparison of theoretical results with different choices of the nuclear average potential. Same conventions as in fig. 1.

deficiency of ref. [1]. The rest of the spectra is not really affected by modifications of the nuclear potential.

For proton spectra (fig. 2), the shift is much less important than for the neutron spectra. First the isospin dependence does not bring any significant modification. The energy dependence introduces a shift of the quasi-elastic peak, the order of which is practically vanishing at 0 degrees and reaches $\sim 15 \mathrm{MeV}$ at 10 degrees (surprisingly a little bit larger for exponentially varying potentials).

The difference of behaviour in neutron and proton spectra can be traced back to the properties of the Fermi seas. In $(p, p)$ reactions (for single scattering) a 
high-energy proton is produced at high energy and small angles (small energy-momentum transfer) after an elastic scattering with a target nucleon. Due to the Pauli principle, this nucleon should be close to the Fermi surface in such a way that (minus) the small momentum transfer brings it out of its Fermi sphere. Although the Fermi momentum has been slightly changed, the available momentum space is not very much affected. Furthermore, the potential energy of the nucleons having been modified by the same amount in the initial and in the final states, this modification has practically no effect, as explained in sect. 2.4. On the other hand, in $(p, n)$ reactions, the emission of a neutron at small energy-momentum transfer implies a collision with a target neutron which, by charge exchange, is transformed into a proton. Due to the Pauli principle, (minus) the small energy-momentum transfer should bring the proton out of the corresponding Fermi sphere. With the standard choice, proton and neutron Fermi momenta are the same and the situation is the same for charge exchange as for quasi-elastic scatterings. With isospin modified potentials, the neutron Fermi momentum is larger than the proton Fermi momentum. At first sight, the Pauli blocking is not very operating in such a case, since the neutron Fermi sphere is noticeably larger than the proton Fermi sphere (at least in heavy nuclei). However, one should not forget that the potentials are such that the Fermi energies are the same for protons and neutrons. Therefore, energy conservation makes only a small part of the Fermi spheres be involved. The net result is however that the relevant parts of the momentum spaces are expected to differ significantly from what they are in the standard case.

Experimentally [18], the position of the quasi-elastic peak is lying at $720 \pm 10 \mathrm{MeV}$ in $p(800 \mathrm{MeV})+{ }^{208} \mathrm{~Pb} \rightarrow$ $n+X$ reactions at 10 degrees. In $p(800 \mathrm{MeV})+{ }^{208} \mathrm{~Pb} \rightarrow$ $p+X$ at 11 degrees, it is situated at $770 \pm 20 \mathrm{MeV}$ [19]. It is thus rewarding to notice that the positions of the peaks are well represented by the simple isospin- and energydependent potentials introduced above. We have to notice however that the amplitude of the quasi-elastic peak is underestimated by the present calculation (using the INCL3 code), whereas it comes out right from calculations with the INCL4 version of the Liège model.

\section{Conclusion}

We have presented a version of the Liège INC model, which accounts for the isospin and energy dependence of the nuclear mean field. The global effects brought by these modifications are not drastic. Nevertheless, some effects are significant and show systematic features. We recall the most important ones. More protons and less neutrons are emitted in the cascade stage. The excitation energy at the end of this stage is increased. As a result, the total proton multiplicity is increased, but the neutron one remains basically the same. The location of the quasi-elastic peak is shifted toward lower energy losses in $(p, n)$ reactions. There is also a shift in the same direction in $(p, p)$ reactions, but it is much smaller. These results are expected to cure remaining deficiencies of the INC model of ref. [1].

We are very grateful to P. Henrotte and B. Van den Bossche for useful discussions.

\section{References}

1. A. Boudard, J. Cugnon, S. Leray, C. Volant, Phys. Rev. C 66, 044615 (2002).

2. J.-J. Gaimard, K.-H. Schmidt, Nucl. Phys. A 531, 709 (1991).

3. A.R. Junghans, M. de Jong, H.G. Clerc, A.V. Ignatyuk, G.A. Kudyaev, K.-H. Schmidt, Nucl. Phys. A 629, 635 (1998).

4. Y. Yariv, Proceedings of the SARE-5 Meeting, edited by D. Filges, F. Goldenbaum, Y. Yariv, (Forschungszentrum Jülich pulication ESS 112-01-T, 2001).

5. J. Cugnon, C. Volant, S. Vuillier, Nucl. Phys. A 620, 475 (1997).

6. T. Ericson, W. Weise, Pions and Nuclei (Clarendon Press, Oxford, 1988).

7. L. Dresner, Oak Ridge report ORNL-TM-193 (1962).

8. P.E. Hodgson, E. Gadioli, E. Gadioli Erba, Introductory Nuclear Physics (Clarendon Press, Oxford, 1997).

9. M.A. Preston, R.K. Bhaduri, Introductory Nuclear Physics (Addison-Wesley, 1995).

10. T. Koopman, Physica 1, 104 (1934).

11. A.H. Wapstra, G. Audi, Nucl. Phys. A 432, 55 (1985).

12. B. ter Haar, R. Malfliet, Phys. Rev. C 36, 1611 (1987).

13. P.E. Hodgson, The Nucleon Optical Potential (World Scientific, Singapore, 1994).

14. J.-P. Jeukenne, A. Lejeune, C. Mahaux, Phys. Rep. C 25, 83 (1976).

15. J.-P. Jeukenne, C. Mahaux, R. Sartor, Phys. Rev. C 43, 2211 (1991).

16. C.H. Johnson, D.J. Horen, C. Mahaux, Phys. Rev. C 36, 2252 (1987).

17. J. Cugnon, C. Volant, S. Vuillier, Nucl. Phys. A 625, 729 (1997).

18. S. Leray et al., Phys. Rev. C 65, 044621 (2002)

19. R.E. Chrien et al., Phys. Rev. C 21, 1014 (1980). 